\title{
COVID-19: Pulse oximeters in the spotlight
}

\author{
Frederic Michard ${ }^{1}(1) \cdot$ Kirk Shelley $^{2} \cdot$ Erwan L'Her $^{3}$
}

Received: 23 May 2020 / Accepted: 17 June 2020 / Published online: 23 June 2020

(c) Springer Nature B.V. 2020

\begin{abstract}
From home to intensive care units, innovations in pulse oximetry are susceptible to improve the monitoring and management of patients developing acute respiratory failure, and particularly those with the coronavirus disease 2019 (COVID-19). They include self-monitoring of oxygen saturation $\left(\mathrm{SpO}_{2}\right)$ from home, continuous wireless $\mathrm{SpO}_{2}$ monitoring on hospital wards, and the integration of $\mathrm{SpO}_{2}$ as the input variable for closed-loop oxygen administration systems. The analysis of the pulse oximetry waveform may help to quantify respiratory efforts and prevent intubation delays. Tracking changes in the peripheral perfusion index during a preload-modifying maneuver may be useful to predict preload responsiveness and rationalize fluid therapy.
\end{abstract}

\section{Introduction}

Pulse oximetry was developed in the 1970's to monitor oxygen saturation $\left(\mathrm{SpO}_{2}\right)$ during anesthesia, and, its inventor, Takuo Aoyagi, sadly passed away on April 18, 2020 at age 84. Although there is no strong evidence that monitoring $\mathrm{SpO}_{2}$ improves outcome in surgical patients [1], pulse oximetry is today a mainstay of perioperative monitoring. It is also widely used in intensive care units (ICU) and emergency departments. In the era of evidence-based medicine, no one is actually questioning its use and clinical value. After all, parachutes have not been scientifically proven to be lifesaving [2] and yet common sense prevails.

In this article we describe recent innovations in pulse oximetry that could be useful in coronavirus disease 2019 (COVID-19) patients.

Frederic Michard

frederic.michard@bluewin.ch

MiCo, Chemin de Chapallaz 4, Denens, Switzerland

2 Professor Emeritus, Department of Anesthesiology, Yale University, New Haven, CT, USA

3 Intensive Care Medicine, University Hospital of Brest, La Cavale Blanche, Brest, France

\section{Self-monitoring of oxygen saturation from home}

Although pulse oximeters have been miniaturized and have become wireless and affordable, they-unlike thermometers - are not yet found in our home medicine cabinet. However, during this pandemic, home pulse oximetry could be very useful in detecting patients requiring oxygen and hospitalization. An initiative led by Peter Pronovost from Cleveland University Hospitals is currently going on for COVID-19 outpatients. This program promotes the use of a finger disposable wireless sensor enabling continuous $\mathrm{SpO}_{2}$ monitoring at home. A dedicated command center receives measurements and alerts via the patient's smartphone. Self-monitoring with miniaturized medical grade pulse oximeters-easily purchased on the web-may be a useful alternative.

\section{Continuous monitoring of oxygen saturation on hospital wards}

The recent surge of hospitalized COVID-19 patients created both material and healthcare worker shortages, raising concerns regarding patient safety. Wireless and wearable sensors are now available to monitor a large number of ward patients at the same time, display the information on a central station, and, in case of clinical deterioration, immediately alert nurses on their pager [3]. During epidemics, wireless sensors would also have the advantage of decreasing the number of physical 
interactions and thereby the risk of virus transmission between healthcare workers and patients [4].

\section{Pulse oximetry for automated oxygen administration}

In patients hospitalized for respiratory failure who require supplemental oxygen, precise manual oxygen titration is difficult to achieve and is time-consuming. Automated oxygen titration devices have been developed to avoid periods of hypoxemia and of hyperoxemia [5]. These systems are based on a closed-loop circuit that enables oxygen flow titration (the output variable) according to the patient's real-time $\mathrm{SpO}_{2}$ (the input value). It intends to reverse the paradigm from a constant oxygen flow with a variable $\mathrm{SpO}_{2}$ value, to a constant target $\mathrm{SpO}_{2}$ set by the physician with continuous oxygen flow variations. The use of such devices has been associated with a greater percentage of time spent within the $\mathrm{SpO}_{2}$ target range, as well as shorter duration of oxygen administration and hospital length-of-stay [6].

\section{Pulse oximetry to quantify respiratory efforts and prevent intubation delays?}

The physiologic response to hypoxemia is an increase in both respiratory frequency and tidal volume. Work of breathing increases, resulting in large changes in pleural pressure which may be responsible for self-inflicted acute lung injury [7]. This phenomenon has been advocated to explain, at least in part, the rapid deterioration of lung function in COVID-19 patients [8]. A very recent study [9] suggests that patients with acute respiratory failure who do not quickly reduce their respiratory effort after initiating non-invasive ventilation will ultimately require tracheal intubation. In this study, respiratory effort was quantified by monitoring the respiratory swings in esophageal pressure. But in clinical practice, esophageal probes are not used in spontaneously breathing patients. In this specific context, the pulsus paradoxus depends mainly on the magnitude of changes in pleural pressure and could be used to approximate respiratory efforts [10]. It can be quantified from a pulse oximetry waveform [11] and is known today as the pleth variability index (PVI). Therefore, when initiating oxygen therapy or non-invasive ventilation, monitoring changes in PVI may help to assess the impact on respiratory efforts and prevent intubation delays.

\section{Pulse oximetry to guide fluid management}

In patients with acute respiratory distress syndrome (ARDS), individualized fluid therapy is desirable to balance the risks of fluid overload (increase in pulmonary edema) with the risks of fluid restriction (decrease in cardiac output and oxygen delivery to the tissues). Predicting preload responsiveness is a way to identify patients who may benefit from fluid administration and, maybe more importantly, to prevent unjustified fluid boluses in preload non-responders. Predicting preload responsiveness may improve outcome in patients with sepsis and septic shock [12] and it was recently recommended by WHO, the Surviving Sepsis Campaign guidelines and the NIH for the hemodynamic management of COVID-19 patients.

The assessment of changes in cardiac output during a passive leg raising maneuver or a lung recruitment maneuver has been proposed to accurately predict preload responsiveness during protective mechanical ventilation [13]. Recent studies have shown that changes in the peripheral perfusion index (PI) - another variable quantified by pulse oximeters-are closely correlated with changes in cardiac output during these brief dynamic maneuvers [14, 15]. Therefore, when cardiac output is not monitored, pulse oximetrythrough the assessment of changes in PI-may also help to predict preload responsiveness and to guide fluid therapy in ARDS patients.

\section{Conclusion}

From home to ICUs, innovations in pulse oximetry go beyond the remote monitoring of $\mathrm{SpO}_{2}$ (Fig. 1) and are susceptible to improve quality of care in patients developing acute respiratory failure, particularly those with COVID-19. 


\section{PULSE OXIMETRY}
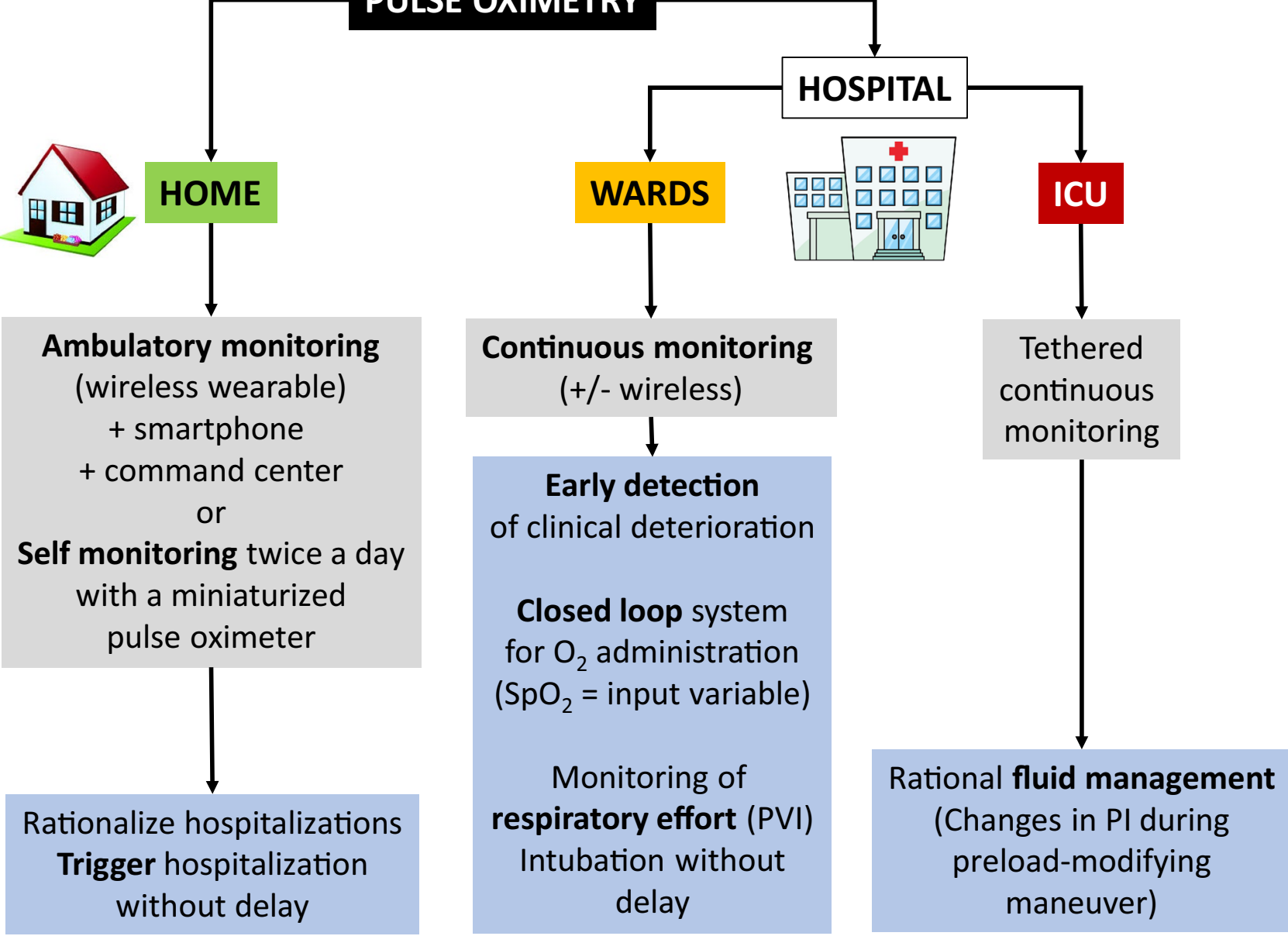

Fig. 1 Innovations in pulse oximetry may help to treat COVID-19 patients from home to the intensive care unit (ICU). PVI pleth variability index; $P I$ perfusion index

\section{Compliance with ethical standards}

Conflict of interest FM is the founder and managing director of MiCo, a Swiss consulting and research firm. MiCo does not sell any medical products and FM does not own shares from any medtech company. KS has no conflict of interest to declare. ELH is co-founder and shareholder of Oxynov Inc., Canada and Consultant for GE Healthcare, Sedana Medical, and Smiths Medical.

\section{References}

1. Shah A, Shelley KH. Is pulse oximetry an essential tool or just another distraction? The role of the pulse oximeter in modern anesthesia care. J Clin Monit Comput. 2013;27:235-42.

2. Smith GCS, Pell JP. Parachute use to prevent death and major trauma related to gravitational challenge: systematic review of randomized controlled trials. BMJ. 2003;327:1459-61.

3. Michard F, Bellomo R, Taenzer A. The rise of ward monitoring: opportunities and challenges for critical care specialists. Intensive Care Med. 2019;45:671-3.
4. Steinhubl SR, Feye D, Levine AC, et al. Validation of a portable, deployable system for continuous vital sign monitoring using a multiparametric wearable sensor and personalized analytics in an Ebola treatment center. BMJ Glob Health. 2016;1:e000070.

5. L'Her E, Dias P, Gouillou M, et al. Automatic versus manual oxygen administration in the emergency department. Eur Respir J. 2017;50:1602552.

6. Denault MH, Péloquin F, Lajoie AC, Lacasse Y. Automatic versus manual oxygen titration in patients requiring supplemental oxygen in the hospital: A systematic review and meta-anaylsis. Respiration. 2019;98:178-88.

7. Brochard L, Slutsky A, Pesenti A. Mechanical ventilation to minimize progression of acute lung injury in acute respiratory failure. Am J Respir Crit Care Med. 2017;195:438-42.

8. Marini JJ, Gattinoni L. Management of COVID-19 respiratory distress. JAMA. 2020. https://doi.org/10.1001/jama.2020.6825.

9. Tonelli R, Fantini R, Tabbi L, et al. Inspiratory effort assessment by esophageal manometry early predicts noninvasive ventilation outcome in de novo respiratory failure: a pilot study. Am J Respir Crit Care Med. 2020. https://doi.org/10.1164/rccm.201912-2512OC.

10. Michard F, Shelley K. Should we monitor pulsus paradoxus via pulse oximetry in COVID-19 patients with acute respiratory failure? Am J Respir Crit Care Med. 2020. https://doi.org/10.1164/ rccm.202004-1504LE 
11. Frey B, Butt W. Pulse oximetry for assessment of pulsus paradoxus: a clinical study in children. Intensive Care Med. 1988;24:242-6.

12. Douglas IS, Alapat PM, Corl KA, et al. Fluid response evaluation in sepsis hypotension and shock: a randomized clinical trial. Chest. 2020. https://doi.org/10.1016/j.chest.2020.04.025.

13. Monnet X, Marik P, Teboul JL. Prediction of fluid responsiveness: An update. Ann Intensive Care. 2016;6:111.

14. Beurton A, Teboul JL, Gavelli F, et al. The effects of passive leg raising may be detected by the plethysmographic oxygen saturation signal in critically ill patients. Crit Care. 2019;23:1-10.
15. de Courson H, Michard F, Chavignier C, et al. Do changes in perfusion index reflect changes in stroke volume during preloadmodifying maneuvres? J Clin Monit Comput. 2019. https://doi. org/10.1007/s10877-019-00445-2.

Publisher's Note Springer Nature remains neutral with regard to jurisdictional claims in published maps and institutional affiliations. 